\title{
Gorillas' (Gorilla g. gorilla) knowledge of conspecifics' affordances: intraspecific social tool use for food acquisition
}

\author{
Jacques Prieur ${ }^{1}$ (D) $\cdot$ Simone Pika ${ }^{2}$
}

Received: 18 July 2019 / Accepted: 13 February 2020 / Published online: 12 March 2020

(c) The Author(s) 2020

\begin{abstract}
The use of tools, long thought to be uniquely human, has now been observed in other animal taxa including several species of birds, non-primate mammals as well as some non-human primate species. Chimpanzees, one of humankind's closest living relatives, exceed all other non-human animal species as they have been reported to use an exceptionally large toolkit. However, relatively little is known about the tool-use skills of the other great ape species. While the majority of tools described are inanimate objects, the use of social tools has received relatively little attention. Here we provide the first evidence of naturally occurring spontaneous exploitative behaviour of a conspecific as a social tool for food acquisition in non-human animals. We observed gorillas in captivity utilising a conspecific as a ladder to gain access to unreachable food. We discuss our findings in the light of other studies on social tool use and suggest the need for more nuanced interpretations of gorillas' cognitive skills.
\end{abstract}

Keywords Social tool use $\cdot$ Great apes $\cdot$ Knowledge of the social world $\cdot$ Gorilla gorilla gorilla $\cdot$ Cognition

\section{Introduction}

The complex use and manufacture of tools has shaped human evolution, culture, and social-cognitive abilities (e.g. Stout 2011; Toth and Schick 2015). Tool use has been defined as "the external employment of an unattached or manipulable attached environmental object to alter more efficiently the form, position, or condition of another object, another organism, or the user itself, when the user holds and directly manipulates the tool during or prior to use and is responsible for the proper and effective orientation of the tool" (Bentley-Condit and Smith 2010, p. 5). Although suggested for several decades as uniquely human (e.g. Oakley

Electronic supplementary material The online version of this article (https://doi.org/10.1007/s10329-020-00805-6) contains supplementary material, which is available to authorized users.

Jacques Prieur

jac.prieur@yahoo.fr

1 Department of Education and Psychology, Comparative Developmental Psychology, Freie Universität Berlin, Habelschwerdter Allee 45, 14195 Berlin, Germany

2 Faculty of Human Sciences, Cognitive BioCognition, Institute of Cognitive Science, University of Osnabrück, Artilleriestrasse 34, 49076 Osnabrück, Germany
1956), tool use has now also been reported in four phyla (Arthropoda, Chordata, Echinodermata, and Mollusca) and nine classes (crabs, insects, spiders, birds, fish, mammals, sea urchins, octopuses, and snails) (e.g. Bentley-Condit and Smith 2010; Shumaker et al. 2011). One of humankind's closest living relatives, chimpanzees (Pan troglodytes), outrival all other non-human animal species, as they use an exceptionally large toolkit for diverse purposes (e.g. Boesch and Boesch 1990; Whiten et al. 2005; McGrew 2013). For instance, chimpanzees have been observed to crack nuts (e.g. Boesch and Boesch 1983), insert linear objects, mostly vegetation, into cavities to extract food items such as ants, honeybees or termites (Goodall 1964; Nishida 1973; Sanz and Morgan 2013), clip leaves in a variety of contexts (e.g. play, courtship, display; Nishida 1980; Kalan and Boesch 2018), and shake, drag or throw objects, usually sticks, in agonistic interactions with conspecifics (e.g. Goodall 1986; Call and Tomasello 2007). In contrast, relatively little is known concerning the tool-use skills of other great ape species, despite long-term observations of populations living in their natural environments (bonobos Pan paniscus: e.g. Kano 1982; Hohmann and Fruth 2003; Furuichi et al. 2015; gorillas Gorilla spp.: e.g. Schaller 1963; Tutin and Fernandez 1983; Breuer et al. 2005; orangutans Pongo spp.: e.g. van Schaik et al. 2003; Russon et al. 2009; Meulman and van 
Schaik 2013). Surprisingly, the majority of tool-use techniques reported so far involve inanimate objects (e.g. sticks, wooden hammers, stones), while relatively little is known about social tool-use abilities.

"Social tool" has been defined as a physical and/or psychological manipulation of an individual to achieve one's own goal (e.g. Bard 1990; Völter et al. 2015). The degree of control between the social tool user and its social tools can vary according to four levels (Völter et al. 2015, 2016). Level 1 represents cases in which the social tool user fully controls its social tool in the same way as for an inanimate entity (e.g. pulling the arm of an individual to access the food that the latter is grabbing). In level 2, the social tool user has only partially physical control of the social tool: self-initiated and self-controlled actions of the social tool are required to achieve the goal (e.g. directing the arm of an individual toward a food item until the social tool has grabbed it, then pulling the arm back). Levels 3 and 4 represent cases of social tool use without any direct physical control. In level 3, the social tool user relies solely on the self-initiated and self-controlled actions of the social tool, who is treated as self-propelled machinery (e.g. giving a tool to an individual who will then act independently of the tool user to get the food item desired by the latter). In level 4 , the social tool user solicits help from the social tool using communicative signalling (e.g. using a pointing gesture to direct the attention of an individual toward a food item to finally obtain it). To date, studies of non-human primates' social manipulation abilities have mainly applied experimental paradigms and employed tasks that require identical and simultaneous or complementary and sequential actions typically of two interactants (e.g. see Völter et al. 2016 for review). These studies showed that several non-human primate species, including three great apes species (bonobos: Hare et al. 2007; chimpanzees: e.g. Crawford 1937; Schweinfurth et al. 2018; orangutans: Völter et al. 2015), tufted capuchin monkeys, (Sapajus apella) (Chalmeau et al. 1997; Mendres and de Waal 2000), and cotton-top tamarins (Saguinus Oedipus) (Cronin et al. 2005; Cronin and Snowdon 2008), socially manipulate conspecifics to achieve their own goals. For instance, Völter et al. (2015) found that orangutan mothers physically manipulated the bodies of their offspring to achieve their own goals (i.e. to obtain high-quality food). Depending on task demands, they exhibited relatively high degrees of flexibility, switching from exploitation to cooperation. In addition, two longitudinal studies focusing on tool use patterns showed social manipulation abilities in western lowland gorillas (Gorilla gorilla gorilla) (Gómez 1990, 1991) and Japanese macaques (Macaca fuscata) (Tokida et al. 1994). Gómez $(1990,1991)$ found that an infant hand-reared gorilla was able to recruit humans to help her obtain unreachable food rewards in a problem-solving task. In particular, the author reported that this infant developed a set of tactile gestures consisting in taking humans by their hand to appropriate locations or taking the hand of humans to external objects she wanted them to manipulate (e.g. latch of a closed door). In a provisioned troop of free-ranging Japanese macaques, three females who previously had learned to insert an inanimate entity (stick) to remove an apple from a horizontal pipe, extended previous experience in handling animate and reliable entities (her infants) to access the food (Tokida et al. 1994). The female macaques pulled their infants out of the pipe after they had caught the food, and one individual even actively pushed her infants into the pipe as though she were inserting a stick. However, so far relatively few reports have provided evidence of social tool use in naturally occurring spontaneous interactions. Studying social manipulations between conspecifics in real-life social contexts (i.e. close to contexts in which natural selection has acted) is very important, since it enables a better understanding of the selection pressures acting upon social manipulation strategies. "Agonistic buffering" is probably the most frequently reported social manipulation behaviour in non-human primates. It refers to male-male interactions in which one male handles a baby and/or a female to reduce the likelihood of aggression (e.g. Assamese macaques, Macaca assamensis: Kalbitz et al. 2017; Barbary macaques, Macaca sylvana: Deag \& Crook 1956; gelada baboons, Theropithecus gelada: Dunbar 1984; olive baboons, Papio anubis: Strum 1984).

Here, we provide the first observations of naturally occurring spontaneous social tool use by non-human animals (gorillas) to obtain access to unreachable food.

\section{Methods}

We observed a group of western lowland gorillas (Gorilla gorilla gorilla) living in the Apenheul Primate Park (Netherlands) from June to July 2017. During the observation period, the group was composed of eight females and five males between 3 and 42 years of age (mean $=13.84$; $\mathrm{SD}=13.36$ ) (see Table 1). For a detailed description of the housing conditions see Prieur 2015.

Table 1 depicts the group composition of the study group as a function of name, age (in years), and sex. The age categories of subjects were based on Breuer et al.'s (2016) definitions for infants ( $0-3$ years), juveniles ( $4-6$ years), and adolescents (7-11 years), and on Stoinski et al.'s (2013) definitions for young (12-20 years) and mature ( $>20$ years) adults (F: female; M: male).

We collected daily behavioural data during four different $1.5 \mathrm{~h}$ sessions per day in the context of a study focusing on gorillas' intraspecific communication signalling. Behaviours were videotaped using a full high-definition video camera (Canon Legria HF M506) equipped with an internal 
Table 1 Individual characteristics of the study group of gorillas

\begin{tabular}{lll}
\hline Name & Age & Sex \\
\hline Mature adult (over 20 years) & 42 & \\
Mintha & 41 & $\mathrm{~F}$ \\
Mandji & 22 & $\mathrm{~F}$ \\
Jambo & & $\mathrm{M}$ \\
Young adult (12-20 years) & 15 & \\
Nemsi & 14 & $\mathrm{~F}$ \\
Gyasi & & $\mathrm{F}$ \\
Adolescent (7-11 years) & 8 & \\
Wimbe & 8 & $\mathrm{M}$ \\
Mapasa & 7 & $\mathrm{M}$ \\
Mfungaji & & $\mathrm{F}$ \\
Juvenile (4-6 years) & 5 & \\
Mzungu & 5 & $\mathrm{M}$ \\
Chama & 5 & $\mathrm{~F}$ \\
Tayari & 5 & $\mathrm{~F}$ \\
Iriki & 5 Infant (0-3 years) \\
Jabari & 3 & $\mathrm{M}$ \\
\hline
\end{tabular}

stereo microphone (sampling rule: focal animal sampling; recording rule: continuous recording; Martin and Bateson 1994). During these systematic observations, we were able to record three instances of an individual seemingly using a conspecific to obtain access to a branch of fresh oak leaves. We describe these three cases and a related tool-use observation in detail in the following paragraph.

\section{Results}

Here, we report four observations of gorillas during the seasonal transition period from spring to summer. In Western Europe, this period is characterised by trees full of fresh leaves with flowers blooming. The gorilla enclosure of the Apenheul Primate Park includes several naturally occurring tree species such as beech (Fagus sylvatica), birch (Betula sp.), and oak (Quercus sp.). As they are attractive sources of food for the folivorous and opportunistically frugivorous western lowland gorillas (Tutin et al. 1991; Remis 1997; Rogers et al. 2004), the trees are protected by wire mesh to protect them against large folivore species. Access to fresh tree leaves, outside regular feeding events, is thus very difficult.

Observation 1 We recorded the first behaviour of interest on 16 June at 01:37 p.m. Underneath an oak tree about 20 $\mathrm{m}$ high with a branch ( $2.5 \mathrm{~m}$ high) of fresh leaves, an infant male, Jabari, was sitting and feeding on a small wooden stick. An adolescent female, Mfungaji, approached Jabari from behind walking quadrupedally and looking at the oak tree branch. She briefly looked back as if to monitor her surroundings and then gently touched and grabbed (for definitions of gestures see Pika et al. 2003) Jabari with both hands. These tactile behaviours lacked effective mechanical force. Mfungaji looked at Jabari's face when she touched and grabbed him, and with a relaxed face (for definitions of facial expressions see Van Hooff 1967; Gold 1991). Jabari also looked at her face and showed an open mouth threat while simultaneously grabbing her left wrist with his left hand. Mfungaji then put her right foot on Jabari's right shoulder. Jabari opened his mouth more widely, thereby revealing his lower canines, and pushed her away using physical force (for differentiation of mechanically effective and ineffective behaviours see Pika 2008). As a result, Mfungaji turned away from Jabari without showing any resistance or opposition. Jabari closed his mouth, now expressing a relaxed face but still using a push gesture without mechanical force. Mfungaji moved even closer to Jabari (approximately $50 \mathrm{~cm}$ ) while picking up the small wooden stick Jabari had previously used to forage. She then stood up in a bipedal posture, supported her own weight with the stick in both hands, and looked up into the out-of-reach oak tree. Jabari was still looking at her. After $3 \mathrm{~s}$, Mfungaji sat down at a distance of $1 \mathrm{~m}$ from Jabari; both were still underneath the oak tree branch (see Electronic Supplemental Material ESM 1 for more details). An adult female, Gyasi, the focus subject of the next two observations, was sitting behind them with her back turned to Mfungaji, so that she could not have seen Mfungaji interacting with Jabari.

Observation 2 We recorded the second behaviour of interest on 17 June 2017 at 10:39 a.m. A juvenile female, Chama, was sitting underneath the same oak tree and feeding on a small oak branch with fresh leaves, which had fallen from the tree. An adult female, Gyasi, approached Chama from behind (see Fig. 1a) and forcefully grabbed her under the shoulders with both hands (see Fig. 1b). She then pulled Chama with both hands (see Fig. 1c), walked beside her, and tried to move her under the oak tree branch $(2.5 \mathrm{~m}$ high) full of fresh leaves. Gyasi showed a tight-lipped face and Chama an open mouth threat. Chama tried to escape by shaking her whole body and tried to bite Gyasi several times (see Fig. 1d). As Gyasi is more powerful than Chama, she forcefully grabbed Chama, thereby immobilising her, and pulled her closer to the branch. Simultaneously, she alternated her gaze between the branch and Chama (see Fig. 1e). In addition, Gyasi adjusted Chama's position so that she was placed directly under the branch in a quadrupedal posture (see Fig. 1f). She grabbed Chama's shoulders with both hands and then put her right foot and subsequently her left foot on Chama's lower back to climb onto her back (see Fig. 1g). Gyasi now stood bipedally on Chama's back, jumped toward the branch (see Fig. 1h), and grabbed it with both hands (see Fig. 1i). She then climbed up into the oak tree and started 

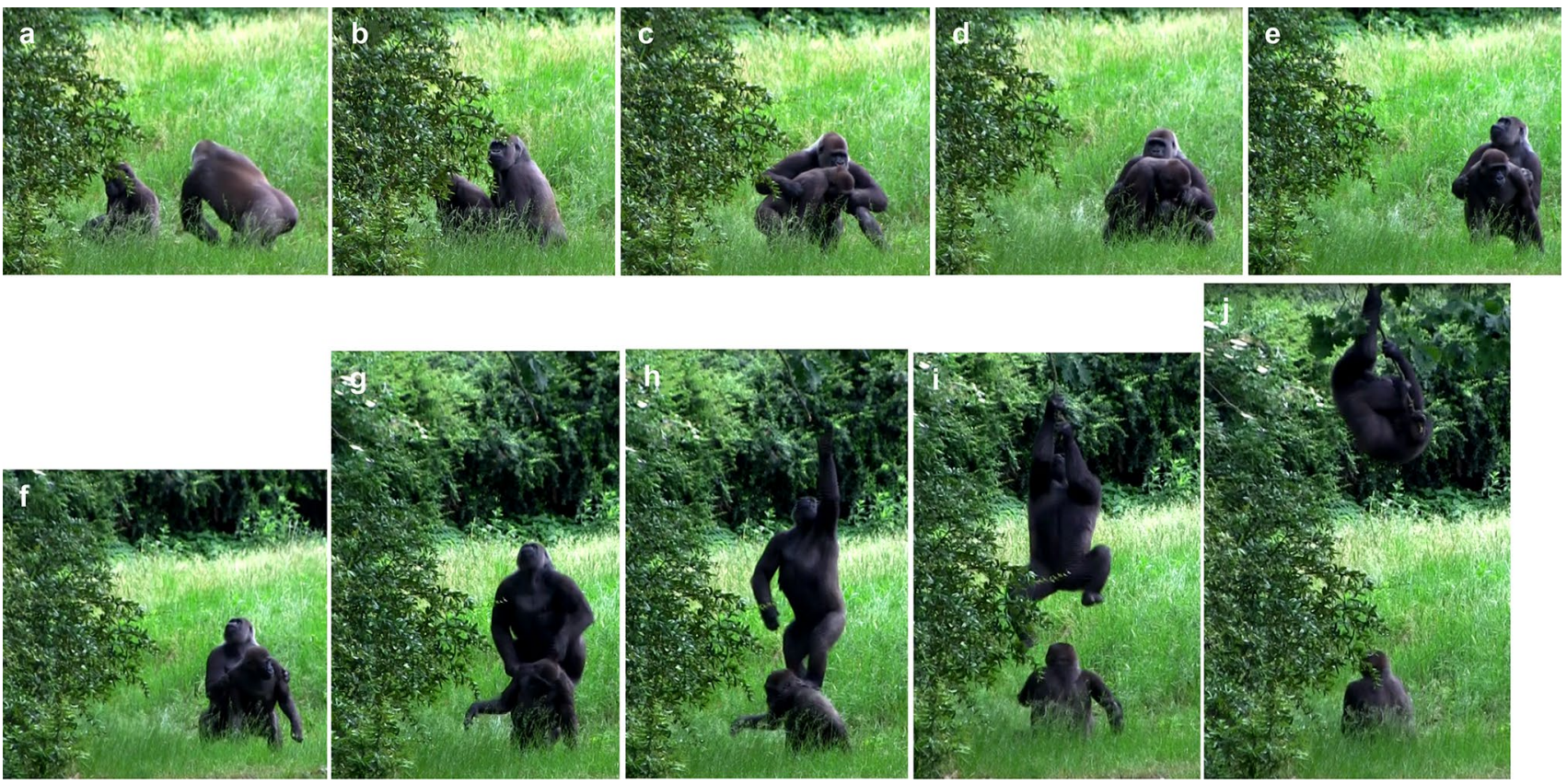

Fig. 1 Behavioural sequence associated with Observation 2. An adult female Gyasi a approached a juvenile female Chama, b forcefully grabbed her under the shoulders with both hands, and $\mathbf{c}$ manipulated her to move her below the oak tree branch. Chama (d) shook her body and bit Gyasi several times. Gyasi (e) continued to firmly

to feed on its leaves (see Fig. 1j; see also ESM 2 for more detail). After a foraging period of approximately $20 \mathrm{~min}$, Gyasi jumped down from the tree without any leaves and hence did not share food with any of the other gorillas, including Chama.

Observation 3 The third behaviour of interest was recorded on 17 June at 01:53 p.m. A mature adult female, Mandji, was lying on the ground underneath the same oak tree. Her daughter, the juvenile female Iriki, approached Mandji from behind and climbed onto her while looking at the oak tree branch, whereas Mandji remained static. Iriki then quickly jumped toward the branch, grabbed it with both hands, and climbed onto the oak tree to get access to fresh leaves while Mandji was looking at her. Iriki fed on leaves in the tree for about $20 \mathrm{~s}$ while collecting several branches with fresh leaves before jumping down the tree (see ESM 3 for more details). Iriki did not share any food with any of the other gorillas, including Mandji.

Observation 4 A related observation was recorded on the same day, 17 June 2017, at 02:40 p.m. The adult female, Gyasi, dragged a wooden log, which she had found approximately $100 \mathrm{~m}$ away, about $1.30 \mathrm{~m}$ and positioned herself underneath the same oak tree branch. Gyasi was in close proximity (ca. $1.5 \mathrm{~m}$ ) to the infant male Jabari, who observed her during the whole subsequent sequence: Gyasi erected the wooden log while firmly grabbing it with both hands (see manipulate Chama while looking at the branch, $\mathbf{f}$ adjusted Chama's position to place her just under the branch, $\mathbf{g}$ climbed onto her back, $\mathbf{h}$ stood bipedally on her and jumped toward the branch, and $\mathbf{i}$ grabbed it with both hands. Eventually, Gyasi (j) climbed up in the oak tree to get food access while Chama was looking at her

Fig. 2a), climbed rapidly onto it while looking at the oak tree branch (see Fig. 2b), jumped toward the branch (see Fig. 2c), grabbed it with both hands (see Fig. 2d), and (e) climbed onto the oak tree to get access to fresh leaves. Attracted by the noise caused by Gyasi, two adolescents, Mfungaji and Wimbe, and one juvenile, Tayari, approached the oak tree branch while observing Gyasi who was climbing up into the tree. Gyasi fed on leaves in the tree for approximately $20 \mathrm{~min}$ before jumping down the tree. Again, she did not bring down any leaves with her and did not share any food with any of her group members.

\section{Discussion}

Here, we provide detailed descriptions of four behavioural events in which a gorilla explored different tactics to gain access to desired and out-of-reach food. Two different explanations may account for these observations.

First, the gorillas may have simply tried to get access to the food in the tree and took opportunistic advantage of conspecifics sitting underneath the oak trees to climb onto them. If this explanation is true, we predicted that gorillas would be frequently located close to the tree. In addition, we predicted that gorillas should show motivation (through gazes and actions) to act in order to achieve their foraging 

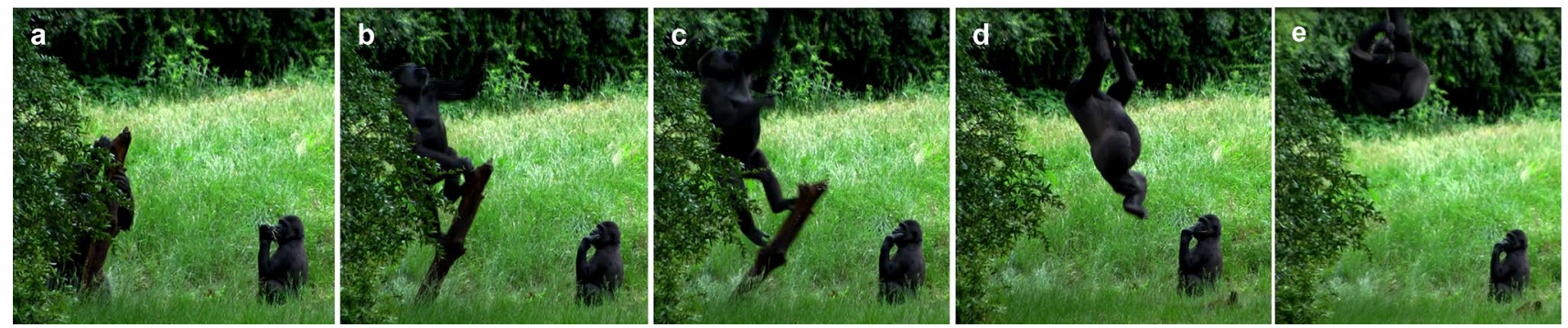

Fig. 2 Behavioural sequence associated with Observation 4. The adult female named Gyasi (on the left) a erected a wooden log under the oak branch while firmly grabbing it with both hands, b climbed onto it while looking at the branch, $\mathbf{c}$ jumped toward the branch, $\mathbf{d}$ grabbed it with both hands, and e climbed onto the oak tree to get access to fresh leaves

Second, the gorillas took into consideration the conspecific's affordance (i.e. conspecific's physical and social characteristics such as his/her strength, size, rank, and/or kinship) to get access to the out-of-reach tree. If this explanation is true, we predicted that strength and/or rank would differ between the two animals involved: the stronger/higherranking animal climbing onto the weaker/lower-ranking one and not vice versa except when kinship was involved, which would favour cooperation and altruism toward genetic kin (e.g. Hamilton 1964; Lehmann and Keller 2006; West et al. 2007). In addition, we predicted that the supporting individual should be strong enough so that climbing onto and standing on his/her back/shoulders would be secure and tall enough to help reach the branch. These predictions are in line with our observations: the initiating individual was physically stronger and higher-ranking than the supporting animal (Observations 1 and 2) except when the individuals were closely related genetically (infant-mother dyad in Observation 3). Furthermore, the manipulated individual had the required strength and size to enable the initiating individual to increase her height and reach the branch (Observations 1-3). However, in Observation 4, the infant male underneath the oak tree branch was not strong or tall enough to help the actor reach the branch, whereas the wooden log was an adequate tool. In a similar vein, other studies report that other great ape species (bonobos: Grueneisen et al. 2017; chimpanzees: Call and Tomasello 2008; Fröhlich et al. 2016; orangutans: Völter et al. 2015), some monkey species (tufted capuchin monkeys: De Waal and Davis 2003; cotton-top tamarins: Cronin et al. 2005; Cronin and Snowdon 2008; long-tailed macaques, Macaca fascicularis: Overduin-de Vries et al. 2014), several non-primate mammals (goats, Capra hircus: Kaminski et al. 2006; hyaenas, Crocuta crocuta: Drea \& Carter 2009), and some species of birds (western scrub-jays, Aphelocoma californica: Dally et al. 2006; keas, Nestor notabilis: Tebbich et al. 1996; Range et al. 2009) take into consideration the social affordances of conspecifics. For instance, chimpanzees are able to adjust their use of gesture in relation to distinct conspecific 
characteristics (age, sex, and kin relationship) (Fröhlich et al. 2016). Keas are able to manipulate conspecifics to gain access to enclosed food in relation to conspecifics' rank status (Tebbich et al. 1996), and the social attention they pay to a conspecific exhibiting food-related behaviours depends on its age (Range et al. 2009). Furthermore, when facing experimental problem-solving tasks, chimpanzees can anticipate actions of dominant conspecifics by understanding the visual perception and knowledge of dominants (e.g. Call and Tomasello 2008; Hare et al. 2000, 2001). Chimpanzees can also choose to recruit the more effective of two conspecific partners based on their previous task success rate (Melis et al. 2006). By using a cooperative-solving task, Tebbich et al. (1996) reported keas' intraspecific coercive instances of dyadic social tool use combining high differential (in strength and/or rank) with low social tolerance. The authors showed that, in dyadic test situations, three dominant keas aggressively approached their respective subordinate partners to force them to operate a lever opening a food apparatus without ever reciprocating. These experiments suggest that rank status would enable them to force "cooperation".

We now discuss our observations focusing especially on current explanations emphasising the emergence and flourishing of diverse complex tool use behaviours in terms of ecological, social, and cognitive factors (e.g. van Schaik et al. 1999; Sanz et al. 2013; Koops et al. 2014). Our Observations 1,2 , and 3 showed individuals interacting with each other in a fundamentally different way depending on the level of control and motivation between the user and the social tool. Observation 3 is linked to the proposed level 1 of social tool use (Völter et al. 2015, 2016; Schweinfurth et al. 2018), with the initiating individual treating the social tool as a physical object (ladder) to reach the branch, while the social tool remained static, thus tolerating this act. Observation 2 is linked to the proposed level 2 of social tool use, with the initiating individual using mechanically effective and coercive behaviours toward the social tool, forcing the latter to perform self-initiated and self-controlled actions so that the initiating individual could climb onto and stand on the social tool's back/shoulders to reach the branch. The initiating individual thus forced the social tool to cooperate, which is different from cooperation occurring upon communicative request that elicits a voluntary response from the social tool (e.g. Yamamoto et al. 2009, 2012). Observation 1 is linked to the proposed level 4 of social tool use, with the initiating individual using no coercive behaviour toward the social tool, and soliciting help from the latter using communicative signaling. However, in Observations 1 and 2, the social tools indicated their refusal to cooperate by performing different types of agonistic communicative behaviours such as an open mouth threat (e.g. van Hooff 1967; Bennett and Fried 1991; Dubois et al. 1991). This is in line with studies showing that gorillas do not seem to cooperate (Harcourt and Stewart 2007; Pelé et al. 2009). Our observations thus provide evidence of different levels of social tool use by gorillas in a foraging context. They also indicate that both interactants' behaviours and physical and social characteristics (strength, size, rank, and/or kinship) may have important role to play in the process of social manipulation. These findings, along with the scarce literature on social tool use (chimpanzees, orangutans, and Japanese macaques: Völter et al. 2015; Schweinfurth et al. 2018; Tokida et al. 1994; keas: Tebbich et al. 1996), suggest that (1) the foraging context may be a useful candidate for investigating social tool use in the lesser-known and under-documented gorillas in terms of socio-cognitive skills, and (2) some features of social tool use might have evolved across different vertebrate lineages (primates and birds) through convergent evolution rather than only phylogenetic continuity. Our findings further emphasise the suggestion by Pika et al. (2019) that "reliable insights into the purpose cognitive abilities serve can only be gained by unravelling specific socio-ecological factors triggering their usage - a task demanding careful, knowledgeable observations of species living in their natural environments." Deeper investigations of the ecological, social, and cognitive bases of social tool use in a wider range of primate species and in other lineages are necessary to improve our understanding of the evolutionary origins of human social manipulation.

\section{Conclusion}

Here, we report the first observations of naturally occurring spontaneous social tool use in a non-human animal species (western lowland gorillas) to obtain access to unreachable food. Our findings reveal that gorillas are able to take into account conspecifics' physical and social affordances (i.e. conspecific's strength, size, rank, and/or kinship), shedding unprecedented light on gorillas' knowledge of their social environment. Although based on only four observations, our results strongly emphasise the need for more nuanced interpretations of gorillas' cognitive skills. In addition, they hopefully will inspire research into gorillas' cognitive skills of individuals living in their natural environments.

Surprisingly, relatively little attention has been given to the comparative evolutionary approach studying social manipulation by human and other animals, despite the significance and permanent impact of manipulation and communication strategies (e.g. manipulation of public opinion through social media, marketing strategies, and consumption patterns) in the development of human societies. We need further research mapping out the phylogeny and ontogeny of social manipulation both within and between socio-ecologically relevant behavioural activities (e.g. foraging) to 
better understand the evolutionary mechanisms underlying the emergence and development of social manipulation.

Acknowledgments Open Access funding provided by Projekt DEAL. We are very grateful to all the keepers and collaborators at the Apenheul Primate Park (Netherlands), and particularly F. Rietkerk for allowing us to study the Apenheul Primate Park's gorilla group. We are also indebted to Ann Cloarec for correcting the English.

Author contributions JP and SP designed the research study on gorilla communication in which the four described observations were recorded. JP collected and analysed the data, and drafted the first manuscript. JP and SP discussed the results and finalised the manuscript.

Funding This study was supported by the Deutsche Forschungsgemeinschaft (DFG, German Research Foundation) project number 407023904 to JP, and by a Sofja-Kovalevskaja Award by the Alexander von Humboldt-Foundation (www.humboldt-foundation.de) to SP.

Data availability The data sets supporting this article have been uploaded as part of the supplementary material.

\section{Compliance with ethical standards}

Conflict of interest The authors declare no competing financial and/or non-financial interests in relation to the work described.

Ethical approval In an effort to avoid influencing the natural behaviour of individuals and group, our study was purely non-invasive, with audio and video recordings taken from a minimum distance of $5 \mathrm{~m}$. The research adhered to the legal requirements of the Netherlands and followed the recommendations of the Animals (Scientific Procedures) Act 1986, as published by the government of the United Kingdom, and the Principles for the 'Ethical Treatment of Non-Human Primates', as stated by the American Society of Primatologists. Permission to conduct research at the Apenheul Primate Park was granted by F. Rietkerk.

Open Access This article is licensed under a Creative Commons Attribution 4.0 International License, which permits use, sharing, adaptation, distribution and reproduction in any medium or format, as long as you give appropriate credit to the original author(s) and the source, provide a link to the Creative Commons licence, and indicate if changes were made. The images or other third party material in this article are included in the article's Creative Commons licence, unless indicated otherwise in a credit line to the material. If material is not included in the article's Creative Commons licence and your intended use is not permitted by statutory regulation or exceeds the permitted use, you will need to obtain permission directly from the copyright holder. To view a copy of this licence, visit http://creativecommons.org/licenses/by/4.0/.

\section{References}

Bard KA (1990) "Social tool use" by free-ranging orangutans: a Piagetian and developmental perspective on the manipulation of an animate object. In: Language and intelligence in monkeys and apes: comparative developmental perspectives, pp 356-378

Bennett C, Fried J (1991) Dallas Zoo-gorilla ethology study. Gorilla behavior advisory group, compilers. Compilation of gorilla ethograms. Atlanta/Fulton County Zoo, Atlanta, pp 7-24
Bentley-Condit VK, Smith EO (2010) Animal tool use: current definitions and an updated comprehensive catalog. Behaviour 147(2):185-132A

Boesch C, Boesch H (1983) Optimisation of nut-cracking with natural hammers by wild chimpanzees. Behaviour 83(3-4):265-286

Boesch C, Boesch H (1990) Tool use and tool making in wild chimpanzees. Folia Primatol 54(1-2):86-99

Breuer T, Ndoundou-Hockemba M, Fishlock V (2005) First observation of tool use in wild gorillas. PLoS Biol 3(11):e380

Call JE, Tomasello ME (2007) The gestural communication of apes and monkeys. Taylor \& Francis Group/Lawrence Erlbaum Associates, Abingdon

Call J, Tomasello M (2008) Does the chimpanzee have a theory of mind? 30 years later. Trends Cognit Sci 12(5):187-192

Chalmeau R, Visalberghi E, Gallo A (1997) Capuchin monkeys, Cebus apella fail to understand a cooperative task. Anim Behav 54(5):1215-1225

Chapman CA, Rothman JM, Lambert JE (2012) Primate foraging strategies and nutrition: behavioral and evolutionary implications. The evolution of primate societies. University of Chicago Press, Chicago, pp 145-167

Crawford MP (1937) The cooperative solving of problems by young chimpanzees. Comp Psychol Monogr 14:1-88

Cronin KA, Snowdon CT (2008) The effects of unequal reward distributions on cooperative problem solving by cottontop tamarins, Saguinus oedipus. Anim Behav 75(1):245-257

Cronin KA, Kurian AV, Snowdon CT (2005) Cooperative problem solving in a cooperatively breeding primate (Saguinus oedipus). Anim Behav 69(1):133-142

Dally JM, Emery NJ, Clayton NS (2006) Food-caching western scrub-jays keep track of who was watching when. Science 312(5780):1662-1665

De Waal FB, Davis JM (2003) Capuchin cognitive ecology: cooperation based on projected returns. Neuropsychologia 41(2):221-228

Doran-Sheehy D, Mongo P, Lodwick J, Conklin-Brittain NL (2009) Male and female western gorilla diet: preferred foods, use of fallback resources, and implications for ape versus old world monkey foraging strategies. Am J Phys Anthropol 140(4):727-738

Drea CM, Carter AN (2009) Cooperative problem solving in a social carnivore. Anim Behav 78(4):967-977

Dubois T, Mead J, Cox C (1991) Lowland gorillas: ethogram (Los Angeles Zoo). Gorilla behavior advisory group, compilers. Compilation of gorilla ethograms. Atlanta/Fulton County Zoo, Atlanta, pp 27-36

Dunbar RIM (1984) Infant-use by male gelada in agonistic contexts: agonistic buffering, progeny protection or soliciting support? Primates 25(1):28-35

Fröhlich M, Wittig RM, Pika S (2016) Play-solicitation gestures in chimpanzees in the wild: flexible adjustment to social circumstances and individual matrices. R Soc Open Sci 3(8):160278

Furuichi T, Sanz C, Koops K, Sakamaki T, Ryu H, Tokuyama N, Morgan D (2015) Why do wild bonobos not use tools like chimpanzees do? Behaviour 152(3-4):425-460

Garber PA (1988) Foraging decisions during nectar feeding by tamarin monkeys (Saguinus mystax and Saguinus fuscicollis, Callitrichidae, Primates) in Amazonian Peru. Biotropica, pp 100-106

Gold KC (1991) Gorilla development study. In: Gorilla Behavior Advisory Group, compilers. Compilation of gorilla ethograms. Atlanta/Fulton County Zoo, Atlanta, pp 52-53

Gómez JC (1990) The emergence of intentional communication as a problem-solving strategy in the gorilla. In: Parker ST, Gibson KR (eds) "Language" and intelligence in monkeys and apes: comparative developmental perspectives. Cambridge University Press, Cambridge, pp 333-355 
Gómez JC (1991) Visual behavior as a window for reading the minds of others in primates. In: Whiten A (ed) Natural Theories of Mind: Evolution, development and simulation of everyday mindreading. B. Blackwell, Oxford, pp 195-207

Goodall J (1964) Tool-using and aimed throwing in a community of free-living chimpanzees. Nature 201(4926):1264

Goodall J (1986) The chimpanzees of Gombe: patterns of behavior. Belknap Press of Harvard University Press, Cambridge

Grueneisen S, Duguid S, Saur H, Tomasello M (2017) Children, chimpanzees, and bonobos adjust the visibility of their actions for cooperators and competitors. Sci Rep 7(1):8504

Hamilton WD (1964) The genetical evolution of social behaviour, I and II. J Theor Biol 7:1-52

Harcourt AH, Stewart KJ (2007) Gorilla society: conflict, compromise, and cooperation between the sexes. University of Chicago Press, Chicago

Hare B, Call J, Agnetta B, Tomasello M (2000) Chimpanzees know what conspecifics do and do not see. Anim Behav 59(4):771-785

Hare B, Call J, Tomasello M (2001) Do chimpanzees know what conspecifics know? Anim Behav 61(1):139-151

Hare B, Melis AP, Woods V, Hastings S, Wrangham R (2007) Tolerance allows bonobos to outperform chimpanzees on a cooperative task. Curr Biol 17(7):619-623

Hohmann G, Fruth B (2003) Culture in bonobos? Between-species and within-species variation in behavior. Current Anthropology 44(4):563-571

Janmaat KR, Polansky L, Ban SD, Boesch C (2014) Wild chimpanzees plan their breakfast time, type, and location. Proc Natl Acad Sci 111(46):16343-16348

Janson CH (1988) Food competition in brown capuchin monkeys (Cebus apella): quantitative effects of group size and tree productivity. Behaviour 105(1-2):53-76

Janson CH, Byrne R (2007) What wild primates know about resources: opening up the black box. Anim Cogn 10(3):357-367

Kalan AK, Boesch C (2018) Re-emergence of the leaf clip gesture during an alpha takeover affects variation in male chimpanzee loud calls. PeerJ 6:e5079

Kalbitz J, Schülke O, Ostner J (2017) Triadic male-infant-male interaction serves in bond maintenance in male Assamese macaques. PLoS One 12(10):e0183981

Kaminski J, Call J, Tomasello M (2006) Goats' behaviour in a competitive food paradigm: evidence for perspective taking? Behaviour 143(11):1341-1356

Kano T (1982) The use of leafy twigs for rain cover by the pygmy chimpanzees of Wamba. Primates 23(3):453-457

Koops K, Visalberghi E, van Schaik CP (2014) The ecology of primate material culture. Biol Lett 10(11):20140508

Lehmann L, Keller L (2006) The evolution of cooperation and altruism-a general framework and a classification of models. J Evol Biol 19(5):1365-1376

Martin P, Bateson P (1994) Measuring behaviour: an introductory guide, 2nd edn. Cambridge University Press, Cambridge

Masi S, Cipolletta C, Robbins MM (2009) Activity patterns of western lowland gorillas (Gorilla gorilla gorilla) at Bai-Hokou, Central African Republic. Am J Prim 71:91-100

McGrew WC (2013) Is primate tool use special? Chimpanzee and New Caledonian crow compared. Philos Trans R Soc B Biol Sci 368(1630):20120422

Melis AP, Hare B, Tomasello M (2006) Chimpanzees recruit the best collaborators. Science 311(5765):1297-1300

Mendres KA, de Waal FB (2000) Capuchins do cooperate: the advantage of an intuitive task. Anim Behav 60(4):523-529

Meulman EJM, van Schaik CP (2013) Orangutan tooluse and the evolution of technology. In: Sanz C, Call J, Boesch B (eds) Tool use inanimals: cognition and ecology. Cambridge University Press, Cambridge, UK, pp 176-202

Nishida T (1973) The ant-gathering behaviour by the use of tools among wild chimpanzees of the Mahali Mountains. J Hum Evol 2(5):357-370

Nishida T (1980) The leaf-clipping display: a newly-discovered expressive gesture in wild chimpanzees. J Hum Evol 9(2):117-128

Noser R, Byrne RW (2007) Mental maps in chacma baboons (Papio ursinus): using inter-group encounters as a natural experiment. Anim Cognit 10(3):331-340

Oakley K (1956) The earliest tool-makers. Antiquity 30(117):4-8

Overduin-de Vries AM, Spruijt BM, Sterck EHM (2014) Long-tailed macaques (Macaca fascicularis) understand what conspecifics can see in a competitive situation. Anim Cognit 17(1):77-84

Pelé M, Dufour V, Thierry B, Call J (2009) Token transfers among great apes (Gorilla gorilla, Pongo pygmaeus, Pan paniscus, and Pan troglodytes): species differences, gestural requests, and reciprocal exchange. J Comp Psychol 123(4):375

Pika S (2008) Gestures of apes and pre-linguistic human children: similar or different. First Lang 28:116-140

Pika S, Liebal K, Tomasello M (2003) Gestural communication in young gorillas (Gorilla gorilla): gestural repertoire, learning, and use. Am J Primatol 60(3):95-111

Pika S, Klein H, Bunel S, Baas P, Théleste E, Deschner T (2019) Wild chimpanzees (Pan troglodytes troglodytes) exploit tortoises (Kinixys erosa) via percussive technology. Sci Rep 9(1):7661

Prieur J (2015) Chimpanzees' and gorillas' intraspecific gestural laterality: A multifactorial investigation (doctorate thesis), Rennes, France: University of Rennes 1 www.theses.fr/en/2015REN1S056

Range F, Horn L, Bugnyar T, Gajdon GK, Huber L (2009) Social attention in keas, dogs, and human children. Anim Cogn 12(1):181-192

Remis MJ (1994) Feeding ecology and positional behavior of western lowland gorillas (Gorilla gorilla gorilla) in the Central African Republic. Ph.D. Thesis. New Haven: Yale University

Remis MJ (1997) Western lowland gorillas (Gorilla gorilla gorilla) as seasonal frugivores: use of variable resources. Am J Primatol 43(2):87-109

Robbins MM, McNeilage A (2003) Home range and frugivory patterns of mountain gorillas in Bwindi Impenetrable National Park, Uganda. Int J Primatol 24(3):467-491

Rogers ME, Maisels F, Williamson EA, Fernandez M, Tutin CE (1990) Gorilla diet in the Lopé Reserve, Gabon: a nutritional analysis. Oecologia 84(3):326-339

Rogers ME, Abernethy K, Bermejo M, Cipolletta C, Doran D, Mcfarland K, Tutin CE (2004) Western gorilla diet: a synthesis from six sites. Am J Primatol 64(2):173-192

Russon AE, van Schaik CP, Kuncoro P, Ferisa A, Handayani DP, Van Noordwijk MA (2009) Innovation and intelligence in orangutans. Geographic variation in behavioral ecology and conservation, Orangutans, pp 279-298

Sanz CM, Call J, Boesch C (eds) (2013) Tool use in animals: cognition and ecology. Cambridge University Press, Cambridge

Schaller GB (1963) The mountain gorilla: ecology and behavior. University of Chicago Press, Chicago

Schweinfurth MK, DeTroy SE, Van Leeuwen EJ, Call J, Haun D (2018) Spontaneous social tool use in chimpanzees (Pan troglodytes). J Comp Psychol 132(4):455

Shumaker RW, Walkup KR, Beck BB (2011) Animal tool behavior: the use and manufacture of tools by animals. JHU Press, Baltimore

Stoinski TS, Lukas KE, Kuhar CW (2013) Effects of age and group type on social behaviour of male western gorillas (Gorilla gorilla gorilla) in North American zoos. Appl Anim Behav Sci 147(3-4):316-323

Stout D (2011) Stone toolmaking and the evolution of human culture and cognition. Philos Trans R Soc Lond B Biol Sci 366(1567):1050-1059 
Strum SC (1984) Why males use infants. In: Taub DM (ed) Primate paternalism. Van Nostrand Reinhold Company, New York

Tebbich S, Taborsky M, Winkler H (1996) Social manipulation causes cooperation in keas. Anim Behav 52(1):1-10

Tokida E, Tanaka I, Takefushi H, Hagiwara T (1994) Tool-using in Japanese macaques: use of stones to obtain fruit from a pipe. Anim Behav 47(5):1023-1030

Toth N, Schick K (2015) Evolution of tool use. In: Muehlenbein MP (ed) Basics in human evolution, Academic Press, pp 193-208

Tutin CEG (1996) Ranging and social structure of lowland gorillas in the Lope Reserve, Gabon. In: McGrew WC, Marchant LF, Nishida T (eds) Great Ape Societies. University of Cambridge Press, Cambridge, pp 58-70

Tutin CE, Fernandez M (1983) Gorillas feeding on termites in Gabon, West Africa. J Mamm 64(3):530-531

Tutin CE, Fernandez M, Rogers ME, Williamson EA, McGrew WC (1991) Foraging profiles of sympatric lowland gorillas and chimpanzees in the Lope Reserve, Gabon. Philos Trans R Soc Lond Ser B Biol Sci 334(1270):179-186

Van Hooff JARAM (1967) The facial displays of catarrhine monkeys and apes. In: Morris D (ed) Primate ethology. Weidenfeld \& Nicolson, London, pp 7-68

Van Schaik CP, Deaner RO, Merrill MY (1999) The conditions for tool use in primates: implications for the evolution of material culture. J Hum Evol 36(6):719-741

Sanz CM, Morgan DB (2013) Ecological and social correlates of chimpanzee tool use. Philos Trans Roy Soc B Biol Sci 368(1630):20120416

Van Schaik CP, Ancrenaz M, Borgen G, Galdikas B, Knott CD, Singleton I, Merrill M (2003) Orangutan cultures and the evolution of material culture. Science 299(5603):102-105

Völter CJ, Rossano F, Call J (2015) From exploitation to cooperation: social tool use in orang-utan mother-offspring dyads. Anim Behav 100:126-134
Völter CJ, Rossano F, Call J (2016) Social manipulation in nonhuman primates: cognitive and motivational determinants. Neurosci Biobehav Rev 82:76-94

Watts DP (1998) Long-term habitat use by mountain gorillas (Gorilla gorilla beringei). 2. Reuse of foraging areas in relation to resource abundance, quality, and depletion. Int J Primatol 19(4):681-702

West SA, Griffin AS, Gardner A (2007) Social semantics: altruism, cooperation, mutualism, strong reciprocity and group selection. J Evol Biol 20(2):415-432

Whiten A, Horner V, De Waal FB (2005) Conformity to cultural norms of tool use in chimpanzees. Nature 437(7059):737

Williamson EA (1988) Behavioural ecology of western lowland gorillas in Gabon. Ph.D. dissertation, University of Stirling, Stirling, $\mathrm{UK}$

Tutin CE, Fernandez M (1985) Foods consumed by sympatric populations of Gorilla g. gorilla and Pan t. troglodytes in Gabon: some preliminary data. Intl J Primatol 6(1):27

Yamamoto S, Humle T, Tanaka M (2009) Chimpanzees help each other upon request. PLoS One 4(10):e7416

Yamamoto S, Humle T, Tanaka M (2012) Chimpanzees' flexible targeted helping based on an understanding of conspecifics' goals. Proc Natl Acad Sci 109(9):3588-3592

Zuberbühler K, Janmaat KRL (2010) Foraging cognition in non-human primates. In: Platt ML, Ghazanfar AA (eds) Primate Neuroethology. Oxford University Press, Oxford, pp 64-83

Publisher's Note Springer Nature remains neutral with regard to jurisdictional claims in published maps and institutional affiliations. 